\title{
Pediatric Kaposi sarcoma in context of the HIV epidemic in sub-Saharan Africa: current perspectives
}

This article was published in the following Dove Press journal:

Pediatric Health, Medicine and Therapeutics

\author{
Nader Kim El-Mallawany ${ }^{1,2}$ \\ Casey L McAtee ${ }^{1,2}$ \\ Liane R Campbell ${ }^{3}$ \\ Peter N Kazembe ${ }^{4,5}$ \\ 'Department of Pediatrics, Section \\ of Hematology and Oncology, Baylor \\ College of Medicine, Houston, TX, \\ USA; ${ }^{2}$ Texas Children's Cancer and \\ Hematology Centers, Houston, TX, \\ USA; ${ }^{3}$ Department of Pediatrics, \\ Baylor College of Medicine \\ Children's Foundation Tanzania, \\ Baylor International Pediatric AIDS \\ Initiative at Texas Children's Hospital, \\ Mbeya, Tanzania; ${ }^{4}$ Department of \\ Pediatrics, Baylor College of Medicine \\ Children's Foundation Malawi, Baylor \\ International Pediatric AIDS Initiative \\ at Texas Children's Hospital, Lilongwe, \\ Malawi; ${ }^{5}$ Department of Pediatrics, \\ Kamuzu Central Hospital, Lilongwe, \\ Malawi
}

\begin{abstract}
The global experience with pediatric Kaposi sarcoma (KS) has evolved immensely since the onset of HIV (human immunodeficiency virus). In this review, current perspectives on childhood KS are discussed in the context of the HIV epidemic in sub-Saharan Africa. Endemic (HIV-unrelated) KS was first described over 50 years ago in central and eastern Africa, regions where human herpesvirus- 8 , the causative agent of $\mathrm{KS}$, is endemic. With the alarming rise in HIV prevalence over the past few decades, KS has become not only the most common HIV-related malignancy in Africa, but also one of the most common overall childhood cancers throughout the central, eastern, and southern regions of the continent. The unique clinical features of pediatric KS that were described in those early endemic KS reports have been re-affirmed by the contemporary experience with HIV-related KS. These characteristics include a predilection for primary lymph node involvement, significant proportions of patients lacking prototypical cutaneous lesions, and the potential for fulminant disease progression. Other clinical features that distinguish childhood KS from adult disease include disease presentation with severe cytopenias, and the common occurrence of childhood KS without severe CD4 count suppression. Distinct clinical heterogeneity in disease presentation and treatment response have been demonstrated. Long-term complete remission and event-free survival can be achieved - especially in children with lymphadenopathic KS - utilizing treatment with antiretroviral therapy plus mild-moderate chemotherapy regimens that are well tolerated, even in low-income settings. A pediatric-specific staging classification and risk-stratification platform have been retrospectively validated, and may help guide therapeutic strategies. With expansion of the HIV treatment infrastructure throughout Africa, coupled with recent developments in establishing comprehensive pediatric oncology programs, there is great potential for improving outcomes for children with KS. Increased awareness of the unique clinical nuances and collaborative evaluations of pediatric-specific treatment paradigms are required to optimize survival for children with $\mathrm{KS}$.
\end{abstract}

Keywords: Kaposi sarcoma, pediatric KS, HHV-8, KSHV, pediatric oncology, global health

\section{Introduction}

Although four epidemiologic variants of Kaposi sarcoma (KS) have been established,

Correspondence: Nader Kim

El-Mallawany

Texas Children's Cancer and Hematology

Centers, Baylor College of Medicine,

Global HOPE (Hematology-Oncology

Pediatric Excellence), I 102 Bates Street,

Feigin Tower, Suite 1030, Houston, TX

77030, USA

Tel +18328247110

Fax +18328251198

Email nader.el-mallawany@bcm.edu childhood KS primarily occurs as either endemic (HIV-unrelated) or epidemic (HIVrelated) disease. Endemic KS was originally described over 50 years ago in eastern and central Africa, ${ }^{1-4}$ the region where the causative agent for KS, human herpesvirus- 8 (HHV-8)/KS-associated herpesvirus (KSHV), is endemic. ${ }^{5,6}$ Decades later, the onset of the HIV epidemic catapulted KS into becoming one of the most common overall childhood cancers in regions of Africa with high HHV-8 and HIV prevalence rates. ${ }^{7,8}$ The two other epidemiologic variants of KS are exceedingly rare in children; classic 
KS primarily occurs in the elderly population of the Mediterranean region, while the occurrence of transplantation-related $\mathrm{KS}$ in children is restricted to case reports. ${ }^{9,10}$ This review will focus on HIV-related pediatric KS in the contextual backdrop of its occurrence in a region where endemic disease was originally described.

The devastation of widespread HIV infection has been most pronounced in sub-Saharan Africa. This imbalance is even more emphatic in children; the 2016 UNAIDS report reveals that $90 \%$ of the world's two million children living with HIV reside in sub-Saharan Africa. The overwhelming prevalence of HIV in a region where HHV-8 is endemic explains the staggering discrepancy in $\mathrm{KS}$ incidence in HIV-infected children globally. ${ }^{11}$ In the pre-antiretroviral therapy (ART) era in the US and Europe, there were merely 14 cases of childhood KS reported over a 20-year span of multiple HIV-cancer registries. ${ }^{12-17}$ In comparison, several reports describing relatively large cohorts of children and adolescents with KS in sub-Saharan Africa have been published in the last decade. ${ }^{18-24}$ Ultimately, KS has become one of the most common pediatric malignancies in central, eastern, and southern Africa. ${ }^{8}$ Increased awareness of its unique clinical characteristics and therapeutic response is vital in the quest to improve overall outcomes for children with cancer throughout the region.

\section{Epidemiology}

In the late 19th and early 20th century, KS existed in the literature as a rare malignancy throughout central-eastern Europe and the Mediterranean region. ${ }^{1}$ The first pediatric case was described in a 5-year-old Italian boy by de Amicis in 1882. ${ }^{82}$ Case reports that followed included children of Asian, European, Middle-Eastern, and African descent, describing a rare but global disease. ${ }^{1,25,26}$

By the middle of the 20th century, it became clear that KS clustered in a belt of countries, moving across the equator from central into southern Africa. ${ }^{3,4,27}$ Even prior to the HIV pandemic, the incidence of KS in Uganda was three times that of Europe, carrying an annual incidence of 3.7 per 100,000 persons..$^{26,28,29}$ In the region stretching across the Democratic Republic of Congo, western Uganda, and Tanzania, lifetime incidence of KS reached as high as 16 per $1,000 . .^{30,31}$

With the arrival of HIV in the late $20^{\text {th }}$ century, the global incidence of KS increased multiple times over. In Uganda, annual incidence of KS in males increased over 10-fold from 1960 to $1990 .{ }^{26}$ Pediatric KS increased dramatically in those regions where KS was previously endemic. ${ }^{32}$ An incidence as high as 59/100,000 has been reported across Botswana,
Malawi, Lesotho, Zimbabwe, Mozambique, South Africa, and Zambia. ${ }^{33}$

Although HIV infection plays a major role, the relative incidence of KS generally corresponds with the seroprevalence of HHV-8, demonstrating significant geographic variation worldwide. ${ }^{30,34-37}$ This should be expected considering the known role of HHV-8 in KS oncogenesis. ${ }^{38} \mathrm{HHV}-8$ is typically transmitted by saliva, and, in the case of children, by food and drink-sharing within households. ${ }^{39}$ In highprevalence areas, seroprevalence increases with age. ${ }^{35,36,40}$ In Uganda, HHV-8 is detected in $23 \%$ of $3-5$ year olds, $32 \%$ of children aged $10-13$, and $50 \%$ of adults over $50 .{ }^{35} \mathrm{HHV}-8$ prevalence is low in children in areas where pediatric KS is rare, such as the US and northern Europe, where HHV-8 is detected in $1-6 \%$ of children. ${ }^{5,41}$ In comparison, HHV-8 prevalence is intermediate in the Mediterranean region. ${ }^{6}$ It is important to note that HHV-8 prevalence varies widely across Africa as well, with the highest rates occurring in central and eastern Africa. ${ }^{30,42}$

Endemic, HIV-negative KS remains prevalent in the regions where it pre-dated HIV. Prevalence of HIV negative patients among pediatric KS cases ranges from $10-14 \%$ in Malawi to $22 \%$ in Uganda. ${ }^{7,22,24,43,44}$ Outside of Africa, HIVunrelated pediatric $\mathrm{KS}$ in the form of transplantation-related and classic disease is extremely rare and has been reported in fewer than 50 patients. ${ }^{45}$

For reasons that are unknown, $\mathrm{KS}$ is more common in males than in females. ${ }^{1,26,34}$ The difference in male versus female incidence is much more pronounced in HIV-negative adults, with a male predominance ranging from 10-20:1. ${ }^{26,34}$ In HIV negative children, the male-to-female ratio ranges from 2-7:1.,22,24,44 The ratio in HIV positive children is tighter, though it still favors males by as much as $2.5: 1$, and may be closer to $1: 1$ in some settings. ${ }^{18,21-23}$ The underlying innate risk factor for endemic KS is as yet unknown, but the male predominance suggests a possible sex-linked genetic predisposition.

Precise and accurate estimation of the disease burden of pediatric KS is challenging due to a paucity of populationbased studies, few hospital registries in endemic regions, under-reporting, and potential misdiagnosis. In a review of pediatric-specific epidemiological studies of KS in Africa, 15 studies published since 1970 were identified. These studies included population-based estimates of pediatric KS, and the reported incidence varied widely. ${ }^{32}$ For example, estimates of KS incidence in Kampala, Uganda through the 1990s ranged from 5-160 cases per 100,000 children, and in Lusaka, Zambia from $0.05-75.7$ cases per 100,000 children during the 
1980s. ${ }^{26,46-48}$ Methods of patient registration and identification of KS cases vary across studies, and comparisons between them should be made with discretion.

The Kampala Cancer Registry of Uganda, established in 1954, offers valuable insight into the incidence of KS before and throughout the HIV epidemic. The registry reported an annual incidence of pediatric KS of 2.5 per million children from 1960-1971, increasing over 20-fold to 55.8 per million children from $1991-1997 .{ }^{26}$ There is some evidence to suggest a decrease in pediatric KS incidence since its peak in the early 1990 s, a phenomenon seen in children within high-income countries as well as in African adults, all likely secondary to increased utilization of ART. ${ }^{49}$ In another study from Kampala, investigators reported a fall in pediatric KS incidence from a peak of 62.6 per million in the early $1990 \mathrm{~s}$ to 44.6 per million in the early $2000 \mathrm{~s} .{ }^{50}$ However, as the pediatric HIV treatment and care infrastructure has expanded, and awareness of pediatric KS has increased, trends have also demonstrated increasing rates of new childhood KS diagnoses, despite increased access to ART in Malawi. ${ }^{51}$ Ultimately, KS is among the top three most-common childhood malignancies overall in numerous countries in central, eastern, and southern Africa, where it carries a disease burden greater than that of childhood acute lymphoblastic leukemia in the US. $8,34,43,50,52$

\section{Clinical characteristics}

From the original reports of endemic KS in Africa, it was clear that the clinical manifestations in children were distinctly different from those seen in adults. ${ }^{3,4}$ Clinical descriptions of adult KS are characterized by widely recognized features - hyperpigmented skin and oral lesions, woody edema, and visceral disease. ${ }^{53,54}$ In contrast, the early descriptions of endemic KS in children offered striking photographic representations of KS presenting primarily with massive lymphadenopathy (Figure 1). ${ }^{3,4}$ The unique features of endemic KS in children included the predominance of lymph node involvement, rapid progression of disease in the absence of treatment, and a significant percentage of patients presenting without prototypical cutaneous lesions., ${ }^{3,4}$

As the contemporary experience with HIV-related pediatric KS in Africa has evolved, it has become increasingly clear that the early descriptions of endemic childhood KS are reproducible decades later. Recently published data from HHV-8 endemic regions with high HIV prevalence demonstrate high rates of lymphadenopathic KS, ranging from $52-93 \%$ in pediatric KS cohorts from Uganda, Malawi, and Mozambique (Table 1). ${ }^{18,19,21-24}$ As the awareness of lymphadenopathic KS has increased, it appears that the

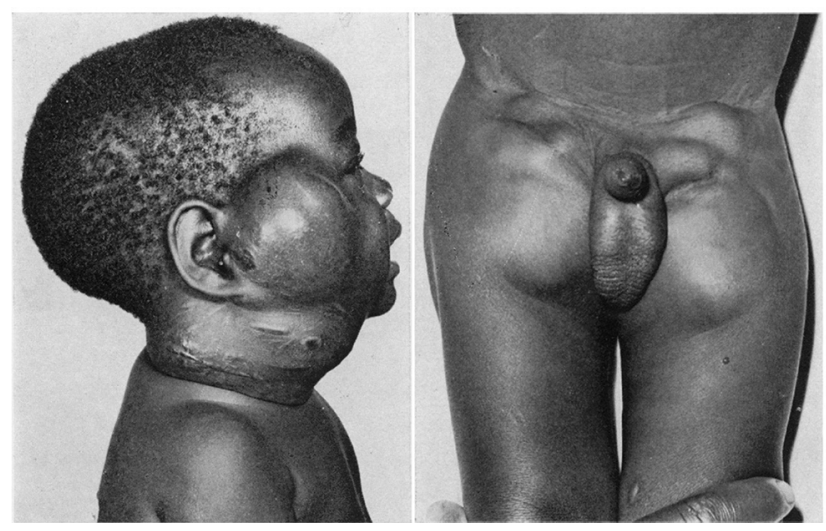

Figure I Photographic representations of lymphadenopathic Kaposi sarcoma. Notes: The typical bulging, massive lymphadenopathy characteristic of lymphadenopathic Kaposi sarcoma is demonstrated in these photographs. Reprinted by permission from Springer Nature: Springer Nature, $\mathrm{Br} J$ Cancer. ${ }^{4}$ Childhood Kaposi's sarcoma: clinical features and therapy, Olweny CL, Kaddumukasa A, Atine I, Owor R, Magrath I, Ziegler JL, @ I 976;33(5):555-560.

recognition of its clinical presentation has resulted in even higher proportions of lymph node disease. The three most recent publications have reported lymphadenopathy to be the most common clinical presentation of childhood KS. ${ }^{22-24}$ It is important to point out that, in the recent cohort from Lilongwe, Malawi, $26 \%$ of all patients presented primarily with lymphadenopathy in the absence of prototypical skin, oral, or woody edema manifestations. ${ }^{23}$ The majority (72\%) of the lymphadenopathy-only patients were diagnosed definitively by biopsy, providing compelling evidence reaffirming the early observations of childhood KS made 50 years ago.

The anatomical sites of involvement in pediatric KS have been previously defined and are described briefly here. ${ }^{23}$ Lymph node $\mathrm{KS}$ is characteristically firm and non-tender; involved nodes tend to be bulging, with diameters greater than $2 \mathrm{~cm}$, and often much larger. They are distinctly different from the shoddy, soft, raisin-sized lymph nodes that are typically seen in the setting of an infectious illness, and/or as a manifestation of HIV-associated generalized lymphadenopathy. Hyperpigmented skin lesions are typically raised papular lesions, but flat macules can also occur. Oral mucosal lesions can be flat or nodular, and, although typically found on the palate, can present anywhere in the oral mucosa. Woody edema is characteristically firm and resembles the texture of tree bark. It typically presents in the extremities, but can occur in the inguinal and pubic region as well. Flesh-colored subcutaneous nodules are characteristically not hyperpigmented, and are located distinctly beneath the skin. They are either movable isolated nodules, or clusters of fibrous lesions associated with underlying woody edema. Some patients present with facial edema that is typically soft, peri-orbital, and distinct from woody edema. Other types of 
Table I Comparison of clinical features among pediatric KS cohorts in Africa

\begin{tabular}{|c|c|c|c|c|c|c|}
\hline Location & Years & $\mathbf{n}$ & Median age & LN involvement & Skin involvement & CD4 count \\
\hline Uganda/Tanzania & $1957-1965$ & 51 & 10 years & $53 \%$ & $53 \%$ & $\mathrm{n} / \mathrm{a}$ \\
\hline Uganda & $1968-1975$ & 12 & 8 years & $83 \%$ & $17 \%$ & $\mathrm{n} / \mathrm{a}$ \\
\hline Uganda & 2004-2007 & 73 & 10.1 years & $60 \%$ & $48 \%$ & median 210 \\
\hline Mozambique & 2003-2008 & 28 & 8.3 years (mean) & $61 \%$ & $82 \%$ & $43 \%$ were 10 \\
\hline Malawi/Botswana & 2003-2009 & 82 & 8 years & $52 \%$ & $83 \%$ & median 297 \\
\hline South Africa & 2005-2009 & 70 & 6.I years (mean) & $30 \%$ & $57 \%$ & mean 440 \\
\hline Blantyre, Malawi & $2009-2012$ & 91 & 8 years (mean) & $73 \%$ & $56 \%$ & median 354 \\
\hline Lilongwe, Malawi & $2010-2013$ & 70 & 8.6 years & $74 \%$ & $59 \%$ & median 368 \\
\hline Mbeya, Tanzania & $2011-2014$ & 34 & I I.5 years & $59 \%$ & $56 \%$ & $76 \%$ were 10 \\
\hline Blantyre, Malawi & $2012-2015$ & 56 & 8 years & $93 \%$ & $93 \%$ & median 40I \\
\hline
\end{tabular}

Abbreviations: KS, Kaposi sarcoma; LN, lymph node; n/a, not applicable; 10, Immune Status 0 in the AIDS Clinical Trial Group TIS staging classification.

lesions that can occur, albeit less frequently, are exophytic or pedunculated masses and hyperpigmented lesions of the eye (conjunctiva).

Visceral disease can present in the lungs or the organs of the abdominal cavity. Because definitive diagnosis is established via bronchoscopy or endoscopy of the gastrointestinal (GI) tract, diagnosing visceral KS can be challenging in low- and middle-income countries where these procedural modalities are lacking. ${ }^{23}$ Pulmonary KS typically presents either with serosanguineous pleural effusions, or with parenchymal disease that can appear as reticulo-nodular infiltrates on a chest X-ray. Patients may present in overt respiratory distress or be asymptomatic; therefore, staging chest X-rays are important to obtain for all patients diagnosed with KS. A high index of suspicion should be maintained in patients with upper airway obstruction that improves after the initiation of chemotherapy, as this may indicate the presence of upper airway KS lesions. Visceral disease in the abdominal cavity presents either in the form of GI disease (with lower GI bleeding) or as severe ascites. Upper GI KS can present with dysphagia, and, in the absence of endoscopy, a high index of clinical suspicion must be maintained in patients who present with dysphagia that improves after the initiation of chemotherapy. ${ }^{23}$ Presentation with visceral disease only, in the absence of any other KS lesions, is relatively difficult to establish in low-income countries.

The clinical signs of visceral KS can overlap with and mimic other opportunistic illnesses. For example, pulmonary tuberculosis and pulmonary KS are challenging to differentiate with limitations in diagnostic resources. Similarly, GI bleeding can occur from both infectious illnesses and GI KS. Therefore, empiric treatment with anti-bacterial, antituberculosis, and anti-parasitic agents is often indicated. In such scenarios, the diagnosis of visceral involvement in children with KS can be a process of exclusion, and is often re-affirmed after failure of these empiric anti-microbial treatments plus apparent improvement in symptoms after initiation of chemotherapy.

Generally, the authors place great emphasis on lymphadenopathic KS in children to increase awareness of this less well-appreciated phenomenon, as missed diagnoses commonly occur. Most general clinicians presume that lymphadenopathy in HIV-infected children is related to tuberculosis or other bacterial infection, a clinical manifestation of lymphoma, or represents HIV-associated generalized lymphadenopathy. Consequently, many patients are eventually diagnosed with lymph node KS after considerable delay - after having initiated ART and having failed empiric anti-bacterial and anti-tuberculosis treatment. Therefore, it is important to maintain a high index of suspicion for the diagnosis of KS in HIV-infected children presenting with persistent lymphadenopathy.

It is likewise important to recognize that childhood $\mathrm{KS}$ can present in the prototypical fashion that is more commonly recognized in adults i.e., with hyperpigmented skin and oral lesions, woody edema, and visceral disease. Hyperpigmented skin lesions are commonly reported, with rates of cutaneous involvement as high as $82-93 \%$ in some cohorts, ${ }^{19,21,24}$ but as low as $48-59 \%$ in others (Table 1). ${ }^{18,22,23}$ Clinical presentation with woody edema tends to occur in older children and adolescents, while lymph node involvement is associated with younger age, especially in the HHV-8 endemic regions of eastern and central Africa. ${ }^{18,21,55}$ Interestingly, data from South Africa, where childhood HHV-8 infection rates are significantly lower than in eastern Africa, ${ }^{30,42}$ revealed a much lower percentage $(30 \%)$ of patients with the lymphadenopathic sub-type, suggesting that regional variation may occur depending on the endemic nature of HHV- $8 .^{20}$

Peripheral blood cytopenias are another unique feature of childhood KS and are relatively common at the time of 
presentation. ${ }^{21-24}$ The extent of such cytopenias can be quite severe, with some patients presenting with life-threatening anemia and/or symptomatic thrombocytopenia. Although peripheral blood count analyses have not been systematically reported in all of the pediatric KS cohorts from Africa, data from Lilongwe and Blantyre, Malawi demonstrate the severity of these cytopenias. In the earlier cohort from Lilongwe, $29 \%$ of patients presented with hemoglobin $<7 \mathrm{~g} / \mathrm{dL}$ and $30 \%$ with platelet count $<100 \times 10^{9} / \mathrm{L}^{21}$ In the more recent cohort, $37 \%$ presented with hemoglobin $<8 \mathrm{~g} / \mathrm{dL}$, while $28 \%$ had a platelet count $<100 \times 10^{9} / \mathrm{L}^{23}$ In the two recently published cohorts from Blantyre, the low end of the interquartile range for platelet count was 66 and $76 \times 10^{9} / \mathrm{L}$, respectively, indicating that at least $25 \%$ of both cohorts presented with significant thrombocytopenia. ${ }^{22,24}$ Although anemia has been reported in African adults with $\mathrm{KS}$, it is rare to find descriptions of severe thrombocytopenia. ${ }^{56}$ Although comprehensive bone marrow evaluations have not been performed in children with KS, the authors theorize that the cytopenias in $\mathrm{KS}$ are not due to malignant infiltration of the marrow, but rather secondary to bone marrow suppression from the systemic effects of KS and the underlying HHV-8 infection. This theory is derived from the analogous phenomenon described in two other HHV-8 related malignancies - multicentric Castleman disease (MCD) and the KSHV inflammatory cytokine syndrome (KICS) - and discussed further in the section on HHV-8 virologic considerations. ${ }^{57-59}$ It is important to point out that these severe cytopenias improved promptly after the initiation of chemotherapy in the recent cohort from Lilongwe, with $88 \%$ of patients having achieved resolution of their severe thrombocytopenia within 2 weeks of the first dose of chemotherapy (without the aid of transfusion). ${ }^{23}$

An additional nuance of childhood KS is that CD4 counts are often not severely suppressed - defying common conceptions of HIV-related disease in adults. A multi-center study from South Africa and the more recent Malawian cohorts have demonstrated median/mean CD4 counts ranging from $354-440$ cells $/ \mathrm{mm}^{3}$, indicating that the majority of patients developed KS despite not having a severely suppressed CD4 count (Table 1). ${ }^{20,22-24}$ The authors hypothesize that intrinsic compromise of qualitative T-cell function in children vertically-infected with HIV render them vulnerable to the oncologic complications of HHV-8 infection, regardless of their quantitative CD4 count. In contrast, adults living in HHV-8 endemic regions will have likely experienced HHV-8 infection during childhood. ${ }^{60}$ In theory, these adults would have already developed an effective immune response to HHV-8 during childhood and would require HIV-induced immune dysfunction to enable the potential for secondary reactivation of HHV-8. This would be mechanistically distinct from an HIV-infected child who experiences primary HHV-8 infection with a naïve and impaired immune system, and potentially contributes to the distinctions between pediatric and adult $\mathrm{KS}$.

\section{Treatment outcomes}

Reports on the treatment of pediatric KS in sub-Saharan Africa have primarily highlighted outcomes for patients receiving chemotherapy plus ART. ${ }^{18-24,61}$ While the success of ART alone for adult KS patients with limited disease has been well documented in the US and Europe, ${ }^{62,63}$ the experience with adult and pediatric KS appears to be different in Africa. ${ }^{64,65}$ Whether this is due to inherent biological differences of KS in Africa, or the tendency for KS in Africa to present as advanced-stage disease, or both, remains unclear. Nonetheless, the authors have observed that pediatric KS in HHV-8 endemic regions uncommonly presents with limited disease. ${ }^{55}$ In reviewing the pediatric $\mathrm{KS}$ data from sub-Saharan Africa, only three of the seven major published cohorts reported any long-term survivors after treatment with ART alone - in two patients out of 73 from Uganda, one out of 70 from the South African cohort, and seven out of 82 from the combined Malawi/Botswana experience (Table 2). ${ }^{18,20,21}$ So, while success has been reported for children with KS treated with ART alone, it is in strikingly few patients, suggesting that the vast majority of children will require chemotherapy plus ART.

A combination of multiple additional factors further contributes to the rationale that ART alone is insufficient therapy for the majority of children with KS. During the early years of the ART experience in Africa, mortality rates reached approximately $60 \%$ (Table 2). ${ }^{18,20-22}$ While the improved outcomes of more recent cohorts (with overall survival in the $60 \%$ range) are potentially rooted in multiple factors, it is important to recognize that the development of systematic KS treatment programs providing both ART and chemotherapy has produced improved outcomes, despite including very sick patients. ${ }^{23,24}$ For example, in a recent pediatric KS cohort from Mbeya, Tanzania, 76\% of patients met criteria for World Health Organization-defined severe immunosuppression at diagnosis, and $38 \%$ had severe acute malnutrition. Despite the complexity of patients in this cohort of 34 children receiving treatment with ART plus chemotherapy, 71\% survived with a median follow-up of 26 months. ${ }^{66}$

Additionally, in the era of increasing availability of ART in sub-Saharan Africa, more recent cohorts have included 
Table 2 Comparison of treatment outcomes among pediatric KS cohorts in Africa

\begin{tabular}{|c|c|c|c|c|c|}
\hline Location & Years & $\mathbf{n}$ & $\begin{array}{l}\text { On ART at time of } \\
\text { KS diagnosis }\end{array}$ & $\begin{array}{l}\text { Approximate } \\
\text { overall survival }\end{array}$ & $\begin{array}{l}\text { Survivors with } \\
\text { ART alone, n (\%) }\end{array}$ \\
\hline Uganda & 2004-2007 & 73 & $11 \%$ & unclear & $2(3)$ \\
\hline Mozambique & 2003-2008 & 28 & $0 \%$ & $71 \%$ & 0 \\
\hline Malawi/Botswana & 2003-2009 & 82 & $27 \%$ & $43 \%$ & $7(9)$ \\
\hline South Africa & 2005-2009 & 70 & $20 \%$ & $40 \%$ & I (I) \\
\hline Blantyre, Malawi & 2009-2012 & 91 & $54 \%$ & $40 \%$ & 0 \\
\hline Lilongwe, Malawi & $2010-2013$ & 70 & $49 \%$ & $58 \%$ & 0 \\
\hline Mbeya, Tanzania & $20 I I-20 \mid 4$ & 34 & $71 \%$ & $71 \%$ & 0 \\
\hline Blantyre, Malawi & $2012-2015$ & 56 & $77 \%$ & $59 \%$ & 0 \\
\hline
\end{tabular}

Abbreviations: KS, Kaposi sarcoma; ART, antiretroviral therapy.

high numbers of patients (ranging from 49-77\%) diagnosed with KS, despite already being on ART (Table 2). ${ }^{22-24,66}$ While some patients develop KS in the scenario of an immune reconstitution inflammatory syndrome phenomenon, ${ }^{67}$ the summarized published experience with HIV-related pediatric KS in Africa demonstrates that ART alone rarely provides adequate treatment. Moreover, the authors anecdotal experience in Malawi and Tanzania re-affirms the early observations of children with endemic KS from 50 years ago - that in the absence of chemotherapy, the majority of patients will experience disease progression, that at times can be fulminant. ${ }^{3,4}$

While various chemotherapy regimens have been utilized in the treatment of pediatric KS in Africa, a universal standard has not been established.$^{61}$ Liposomal doxorubicin is widely accepted as the standard first-line chemotherapeutic option for the treatment of adult KS in high-income countries; however, due to its prohibitive cost, it is not available in low-income settings in Africa. ${ }^{60,64,65}$ The chemotherapeutic agents that have been described for the treatment of pediatric $\mathrm{KS}$ in Africa include bleomycin, vincristine, standard doxorubicin, etoposide, and paclitaxel-either as monotherapy or in various combinations. ${ }^{18-24}$ As previously mentioned, the more recent cohorts have reported improved treatment outcomes, but the better survival rates are probably attributable to multiple contributing factors rather than inherent differences in the chemotherapy regimens. Improvements in the overall pediatric HIV treatment infrastructure, supportive care capacity, ancillary services that support the diagnosis and treatment of childhood cancer in Africa, and increased awareness of the clinical presentation of pediatric KS are among the important factors that have contributed to overall improved outcomes. While a comparison of heterogeneous cohorts across Africa and the various treatment outcomes reported may not agree on a single universal treatment approach, there is a common need to identify safe but effective regimens that minimize the treatment toxicity in complex immunosuppressed patients. ${ }^{61}$
The original chemotherapeutic approach to KS in Malawi was vincristine monotherapy; this decision was based upon the limited availability of chemotherapy, as well as the safety profile of a non-myelosuppressive agent like vincristine in the setting of limited capacity to deliver complex oncology care in HIV-infected patients. However, the early experience with vincristine monotherapy demonstrated its relative inefficacy and resulted in the search for improved chemotherapeutic options, leading to the combination regimen of bleomycin and vincristine (BV). ${ }^{22,68} \mathrm{BV}$ offered a reasonable and practical alternative, being a minimally myelosuppressive regimen that could be administered in an outpatient setting without the extensive supportive care resources that are commonly required for the majority of chemotherapy regimens utilized in pediatric oncology centers in highincome countries. Based upon the favorable experience with BV early on, it became the standard regimen for the pediatric KS treatment programs in Lilongwe, Malawi and Mbeya, Tanzania. ${ }^{21,66}$

As the pediatric KS experience in Lilongwe and Mbeya evolved, it became apparent that, while BV was safe and produced excellent long-term outcomes in some patients, it was inadequate to prevent KS-related mortality in others. ${ }^{21}$ A retrospective evaluation of the clinical factors associated with event-free survival (EFS) and overall survival (OS) in 70 pediatric KS patients in Lilongwe determined distinct findings. ${ }^{23} \mathrm{~A}$ significant favorable association was observed between lymphadenopathic KS and both improved EFS and OS. ${ }^{23}$ This contrasted dramatically with children having visceral KS or disseminated skin/oral disease (defined as having $\geq 20$ hyperpigmented skin/oral lesions with widespread distribution), who demonstrated an increased risk for mortality and a failure to achieve EFS. ${ }^{23}$ Woody edema was associated with increased risk for failure to achieve EFS, but without increased mortality. ${ }^{23}$ Overall, BV was well tolerated, with $18.6 \%$ of patients developing grade 4 neutropenia, and without incidence of grade 3 or 4 thrombocytopenia - an 
important factor for clinical sites with limited access to platelet transfusions. The overall treatment-related mortality was $7 \%$, which compared favorably to the $10 \%$ treatmentrelated mortality among all pediatric oncology patients in Lilongwe, establishing BV as a relatively safe first-line chemotherapeutic option. ${ }^{23,43}$

Based upon the spectrum of distinct clinical phenotypes observed in pediatric KS patients, a risk-stratification platform was proposed with the ultimate goal of guiding treatment strategies. The Lilongwe Pediatric KS Staging Classification was derived from the aforementioned analyses of clinical risk factors differentiating four clinical groups with distinct prognostic patterns after treatment with BV plus ART (Figure 2): Stage 1, mild KS limited to cutaneous and oral manifestations (expected to have favorable survival, potentially without requiring chemotherapy); stage 2, lymphadenopathic KS (2-year EFS 73\% and OS 75\%); stage 3 , woody edema KS (2-year EFS 29\% and OS 79\%); and stage 4, visceral and/or disseminated skin/oral KS (2-year EFS $0 \%$ and OS 12\%). ${ }^{55}$ This classification ultimately seeks to determine risk-stratified therapeutic options-guiding which patients may be treated with ART alone, those who may have a favorable prognosis with BV plus ART, and those who require alternative chemotherapeutic options due to the high risk of treatment failure with BV. ${ }^{55}$ Ultimately, pediatric KS is similar to essentially all childhood malignancies, demonstrating distinct heterogeneity in clinical features and treatment response, thereby necessitating a risk-stratified treatment approach to individualize therapeutic options based upon expected prognostic criteria.

\section{Virologic considerations}

While a comprehensive review of HHV-8 viral pathophysiology is beyond the scope of this paper, one cannot discuss KS without addressing the essential role of the causative agent. For further in-depth discussion of this topic, the reader is referred to recently published expert reviews on HHV-8 and its associated malignancies. ${ }^{60,69,70} \mathrm{HHV}-8$ was first discovered in KS tissue biopsies in the $1990 \mathrm{~s},{ }^{38}$ and subsequently has also been associated with several lymphoproliferative disordersprimary effusion lymphoma (including the extracavitary variant), $\mathrm{MCD}$, diffuse large B-cell lymphoma arising in the setting of MCD, and germinotrophic lymphoproliferative disorder. ${ }^{71}$ It is important to note that HHV-8 associated lymphoproliferative disorders can occur concurrently with $\mathrm{KS} .{ }^{72} \mathrm{KICS}$ is an additional recently defined disorder that is typically associated with KS or primary effusion lymphoma and is characterized as a hyper-inflammatory state caused by excessive levels of various cytokines, especially interleukin (IL)-6 and IL-10. ${ }^{58,73}$ The HHV-8 related viral pathophysiology in KICS is analogous to that seen in MCD, and the two conditions share significant clinical and biological overlap. ${ }^{59}$

A distinguishing factor that is common to both MCD and KICS is the role of lytic phase HHV-8 replication in driving the disease pathophysiology. ${ }^{59}$ In contrast, adult KS in the US and Europe generally arises in the setting of latent phase HHV-8 infection. ${ }^{74,75} \mathrm{~A}$ unique aspect of lytic phase infection is expression of the HHV-8 encoded protein viral IL-6, a homolog to human IL-6. ${ }^{76,77}$ Elevated levels of both viral and human IL-6 are characteristic of MCD and KICS, and these excessive cytokine levels play an important role in the unique

Stage 1, Mild and moderate cutaneous/oral KS:
1A, Mild: Disease limited to hyperpigmented skin lesions, flat oral mucosal lesions, and/or
flesh colored subcutaneous nodules, total < 10 lesions.
1B, Moderate: Having any of the following features, alone or in combination: a total of $10-19$
hyperpigmented skin/oral lesions, nodular oral involvement, conjunctivaleye involvement,
exophyticmass, or any patient not meeting the criteria for stages $1 \mathrm{~A}, 2,3$, and 4 .
Stage 2, Lymphadenopathic KS: Having lymph node involvement, with or without any of the
criteria for stage 1.
Stage 3, Woody edema KS: Woody edema with or without any of the criteria for stages 1 and 2
3A: edema involving $<10 \%$ of estimated body surface area (BSA)
3B: edema involving $>10 \%$ of estimated BSA
BSA estimates performed using the wallace rule of 9 's utilized in burn victims
Stage 4, Visceral and/or disseminated cutaneous/oral KS: Clinical* pulmonary or abdominal
visceral involvement and/or having $\geq 20$ hyperpigmented skin/oral lesions in a widespread
distribution (excluding flesh colored subcutaneous nodules), with or without criteria for stages
1, 2 and 3 . Coalescing or confluent hyperpigmented skin lesions localized to an anatomic
region count as one lesion per cluster.

Figure 2 The modified Lilongwe pediatric Kaposi sarcoma staging classification.

Notes: *See "Clinical characteristics" section for descriptions of clinical pulmonary or abdominal visceral involvement in resource-limited settings in the absence of bronchoscopy and endoscopy. Data from El-Mallawany et al. ${ }^{55}$

Abbreviation: KS, Kaposi sarcoma. 
clinical manifestations of both conditions. ${ }^{58,59,73,78}$ Peripheral blood cytopenias are common in both MCD and KICS, and appear to occur as a result of viral and cytokine-mediated bone marrow suppression. ${ }^{57}$ Analysis of bone marrow findings in patients with MCD and cytopenias reveal a distinct lack of malignant infiltrates in the marrow, and a lack of evidence for a hemophagocytic process. ${ }^{57}$ Common findings in the bone marrow evaluations included reactive plasmacytosis and scattered HHV-8 infected mononuclear cells. ${ }^{57}$

As an HHV-8 associated malignancy with clinical manifestations that commonly include lymphadenopathy and peripheral blood cytopenias, MCD shares clinical overlap with the common findings of lymphadenopathy and cytopenias seen in pediatric $\mathrm{KS}$ patients in $\mathrm{HHV}-8$ endemic regions of Africa. Although all of the lymph node biopsies in pediatric $\mathrm{KS}$ patients from Lilongwe have revealed only $\mathrm{KS}$, without evidence of $\mathrm{MCD},{ }^{23}$ the authors hypothesize that it is only a matter of time before increased access to pathology resources in the region will enlighten a broader understanding of the spectrum of HHV-8 associated malignancies in endemic regions. Indeed, a case series of adults with MCD from Lilongwe was recently published, establishing an important precedent to increase awareness of this diagnostic entity. ${ }^{79}$ Moreover, a study evaluating the HHV-8 viral profile from Malawian adult KS specimens demonstrated the existence of a sub-group of patients who exhibited an extended viral transcription profile, in comparison to adult KS from the US, which is characterized by transcripts restricted to the HHV-8 latency locus. ${ }^{80}$ Synthesizing these considerations-the overlap in clinical features between pediatric KS and other HHV-8 associated lymphoproliferative disorders that are typically driven by lytic phase infection, plus the established precedence of a distinct viral pathophysiology in adult KS in Africa - the authors hypothesize that the unique features of childhood KS in HHV-8 endemic regions may be rooted in distinct viral pathophysiologic mechanisms.

\section{Future directions}

Over the past half-century, childhood KS has become increasingly relevant in sub-Saharan Africa. As one of the most common overall childhood cancers in numerous countries in central, eastern, and southern Africa, there is a definite need for improved therapeutic strategies, especially with expanding pediatric HIV and oncology treatment infrastructure throughout the region. ${ }^{81}$

Based upon feedback received for the original proposed paradigm for the Lilongwe Pediatric KS Staging Classification, we have recently made a slight modification to the staging system (Figure 2). In the original classification, stage 1 was intended to include patients with mild cutaneous/oral disease, a group that might be expected to improve with ART alone. This meant that stage 2 included both patients with moderate cutaneous/oral disease, plus those with KS lymphadenopathy (as both groups require chemotherapy plus ART). This caused confusion in interpreting the staging classification, as stage 2 included a broad list of clinical features. Therefore, in an effort to render the staging classification easier to define, the modified classification restricts stage 2 to patients with KS lymphadenopathy. Thus, both patients with mild and moderate cutaneous/oral disease are assigned to stage 1 , as stages $1 \mathrm{~A}$ and $1 \mathrm{~B}$, respectively.

In re-analyzing the data based upon this modification, it is apparent that the outcomes reported in the pediatric KS staging classification/risk stratification paper remain unchanged. Of the 37 patients originally identified as stage 2 in the published data, 34 (92\%) were assigned to stage 2 because of lymph node involvement; three patients would have been stage $1 \mathrm{~B}$ using the modified system. In re-analyzing survival outcomes for the modified stage 2 group of patients with KS lymphadenopathy, the 2-year EFS is $74 \%$, similar to the 2-year EFS of $73 \%$ in the publication. ${ }^{55}$ This modified staging classification provides a clearer differentiation of the clinical phenotypes - (1) KS limited to cutaneous/oral involvement, (2) lymphadenopathic KS, (3) woody edema KS, and (4) visceral and/or disseminated $\mathrm{KS}$.

The current therapeutic approach for the pediatric KS treatment programs in Lilongwe, Malawi and Mbeya, Tanzania involves a risk-stratified, response-adapted regimen based upon the pediatric KS staging classification and subsequent response to treatment (Figure 3). ${ }^{55}$ As the experience in Malawi has demonstrated, response to treatment is important to monitor. ${ }^{23}$ This led to a new treatment response definition, termed induction failure, which referred to the failure to achieve at least a $90 \%$ reduction in size of all $\mathrm{KS}$ lesions (based upon subjective assessment) after the initial induction phase of BV chemotherapy (four cycles given every 2 weeks). Although it is expected for stage 3 woody edema to exhibit a slow response to chemotherapy ( $83 \%$ induction failure rate), for all patients without woody edema, failure to respond robustly to induction BV appears to be a poor prognostic sign and an indication to intensify therapy. Induction failure carried an Odds Ratio of 6.21 (95\% Confidence Interval=1.93-19.99, $P$-value $<0.01)$ for failure to achieve EFS, and an Odds Ratio of 3.02 (95\% Confidence Interval $=0.95-9.65, P$-value $=0.06)$ for risk of death. ${ }^{23}$ Induction failure potentially represents a concerning prognostic sign that may predict an unfavorable 


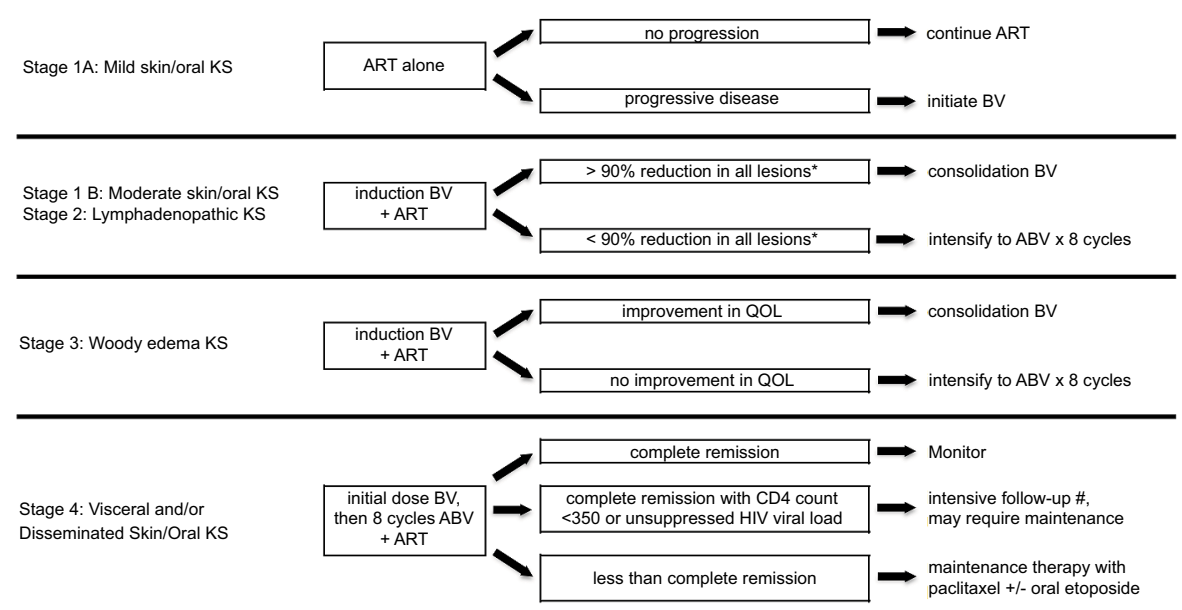

Figure 3 Treatment design schema for a risk-stratified and response-adapted therapeutic approach to pediatric Kaposi sarcoma.

Notes: *Subjective determination of percent decrease in size of all lesions; \# stage 4 patients achieving complete remission who have a persistently low CD4 count $<350$ or unsuppressed HIV viral load are at high risk for relapse and should be monitored closely (ideally every 2 weeks for 3 months). Please note that, for patients with endemic (HIV-unrelated KS), ART is not included in the therapeutic approach, and therefore patients with stage IA disease would be treated the same as patients with stage IB KS. Data from El-Mallawany et al. ${ }^{23,55}$

Abbreviations: KS, Kaposi sarcoma; ART, antiretroviral therapy; progressive disease is defined as an increase in the size of existing lesions or the appearance of new lesions; BV, = bleomycin and vincristine; induction BV includes four cycles of BV given every 2 weeks, consolidation BV includes four cycles of BV given every 4 weeks; ABV, doxorubicin, bleomycin, and vincristine, QoL, quality-of-life; complete remission is defined as no clinical evidence of KS lesions; HIV, human immunodeficiency virus.

outcome for patients without woody edema. Hence intensification to doxorubicin, bleomycin, and vincristine $(\mathrm{ABV}) \times 8$ cycles is indicated for stage $1 \mathrm{~B}$ and stage 2 patients with induction failure, as well as those who experience disease progression or relapse (not due to primary failure of HIV suppression). For those patients who experience worsening of their KS due to failure to control HIV, prompt adjustments in the ART regimen are critical. It is important to point out, though, that woody edema KS (stage 3 ) is characterized as an indolent, almost chronic condition, with low mortality from KS itself. Therefore, intensification of chemotherapy is not indicated, but rather a focus on improving quality-of-life for patients who typically live with stable chronic edema after completing chemotherapy.

Ultimately, future guidelines that can be adapted across the diverse regions of Africa and worldwide are needed. A prospective evaluation of this risk-stratified, response-adapted approach to therapy in a multi-center international trial is required to determine the optimal therapeutic approach to pediatric KS. In the meantime, the authors advocate for increased awareness of the unique clinical features seen in the pediatric population in an effort to expand access to effective treatment regimens and improve overall outcomes for patients across the region.

\section{Acknowledgments}

The authors express gratitude and admiration to the many patients and families battling against the injustice of poverty and the misfortune of severe illness. We thank the many brilliant individuals who have contributed to the development of the pediatric KS treatment programs across the clinical network of sites in Africa set up by the Baylor College of Medicine International Pediatric AIDS Initiative at Texas Children's Hospital. Especially at the sites in Lilongwe, Malawi and Mbeya, Tanzania, we recognize the expert clinical care and guidance of William Kamiyango, Jimmy Villiera, and Jason Bacha, all of whom made immense contributions to developing the current treatment paradigm. We also gratefully acknowledge the external support of many individuals who have helped guide the clinical programs over the past decade, including Parth Mehta, Jeremy Slone, Carrie Cox, Carrie Kovarik, Michael Scheurer, Carl Allen, Joseph Lubega, Gordon Schutze, Mark Kline, David Poplack, Dirk Dittmer, Asulwisye Kapesa, Phoebe Nyasulu, Avni Bhalakia, Maria Kim, and Saeed Ahmed. We extend sincere gratitude to the many colleagues working at the Baylor Children's Foundation Clinical Centres of Excellence in Lilongwe, Malawi and Mbeya, Tanzania, the Tingathe Outreach Program, and Kamuzu Central Hospital.

\section{Disclosure}

The authors report no conflicts of interest in this work.

\section{References}

1. Dutz W, Stout AP. Kaposi's sarcoma in infants and children. Cancer. 1960;13:684-694.

2. Davies JN, Lothe F. Kaposi's sarcoma in African children. Acta Unio Int Contra Cancrum. 1962;18:394-399.

3. Slavin G, Cameron HM, Forbes C, Mitchell RM. Kaposi's sarcoma in East African children: a report of 51 cases. J Pathol. 1970;100(3): 187-199.

4. Olweny CL, Kaddumukasa A, Atine I, Owor R, Magrath I, Ziegler JL. Childhood Kaposi's sarcoma: clinical features and therapy. Br JCancer. 1976;33(5):555-560. 
5. Martro E, Bulterys M, Stewart JA, et al. Comparison of human herpesvirus 8 and Epstein-Barr virus seropositivity among children in areas endemic and non-endemic for Kaposi's sarcoma. J Med Virol. 2004;72(1):126-131.

6. Gao SJ, Kingsley L, Li M, et al. KSHV antibodies among Americans, Italians and Ugandans with and without Kaposi's sarcoma. Nat Med. 1996;2(8):925-928.

7. Ziegler JL, Katongole-Mbidde E. Kaposi's sarcoma in childhood: an analysis of 100 cases from Uganda and relationship to HIV infection. Int J Cancer: 1996;65(2):200-203.

8. Stefan DC. Patterns of distribution of childhood cancer in Africa. JTrop Pediatr. 2015;61(3):165-173.

9. Camcioglu Y, Picard C, Lacoste V, et al. HHV-8-associated Kaposi sarcoma in a child with IFNgammaR1 deficiency. J Pediatr. 2004;144(4): 519-523.

10. Le J, Gantt S, AST Infectious Diseases Community of Practice. Human herpesvirus 6, 7 and 8 in solid organ transplantation. Am J Transplant. 2013;13(Suppl 4):128-137.

11. Pediatric Aids-Defining Cancer Project Working Group for IeDEA Southern Africa, TApHOD, COHERE in EuroCoord, et al. Kaposi sarcoma risk in HIV-infected children and adolescents on combination antiretroviral therapy from sub-Saharan Africa, Europe, and Asia. Clin Infect Dis. 2016;63(9):1245-1253.

12. Biggar RJ, Frisch M, Goedert JJ. Risk of cancer in children with AIDS. AIDS-Cancer Match Registry Study Group. JAMA. 2000;284(2) 205-209.

13. Pollock BH, Jenson HB, Leach CT, et al. Risk factors for pediatric human immunodeficiency virus-related malignancy. JAMA. 2003;289(18):2393-2399.

14. Kest H, Brogly S, McSherry G, Dashefsky B, Oleske J, Seage GR, 3rd. Malignancy in perinatally human immunodeficiency virus-infected children in the United States. Pediatr Infect Dis J. 2005;24(3):237-242.

15. Granovsky MO, Mueller BU, Nicholson HS, Rosenberg PS, Rabkin CS. Cancer in human immunodeficiency virus-infected children: a case series from the Children's Cancer Group and the National Cancer Institute. J Clin Oncol. 1998;16(5):1729-1735.

16. Caselli D, Klersy C, de Martino M, et al. Human immunodeficiency virus-related cancer in children: incidence and treatment outcomereport of the Italian Register. J Clin Oncol. 2000;18(22):3854-3861.

17. Evans JA, Gibb DM, Holland FJ, Tookey PA, Pritchard J, Ades AE. Malignancies in UK children with HIV infection acquired from mother to child transmission. Arch Dis Child. 1997;76(4):330-333.

18. Gantt S, Kakuru A, Wald A, et al. Clinical presentation and outcome of epidemic Kaposi sarcoma in Ugandan children. Pediat Blood Cancer. 2010;54(5):670-674.

19. Vaz P, Macassa E, Jani I, et al. Treatment of Kaposi sarcoma in human immunodeficiency virus-1-infected Mozambican children with antiretroviral drugs and chemotherapy. Pediatr Infect Dis J. 2011;30(10):891-893.

20. Stefan DC, Stones DK, Wainwright L, Newton R. Kaposi sarcoma in South African children. Pediatr Blood Cancer. 2011;56(3):392-396.

21. Cox CM, El-Mallawany NK, Kabue M, et al. Clinical characteristics and outcomes of HIV-infected children diagnosed with Kaposi sarcoma in Malawi and Botswana. Pediatr Blood Cancer. 2013;60(8): 1274-1280.

22. Chagaluka G, Stanley C, Banda K, et al. Kaposi's sarcoma in children: an open randomised trial of vincristine, oral etoposide and a combination of vincristine and bleomycin. Eur J Cancer. 2014;50(8):1472-1481.

23. El-Mallawany NK, Kamiyango W, Slone JS, et al. Clinical factors associated with long-term complete remission versus poor response to chemotherapy in HIV-infected children and adolescents with Kaposi sarcoma receiving bleomycin and vincristine: a retrospective observational study. PloS One. 2016;11(4):e0153335.

24. Macken M, Dale H, Moyo D, et al. Triple therapy of vincristine, bleomycin and etoposide for children with Kaposi sarcoma: Results of a study in Malawian children. Pediatr Blood Cancer. 2018;65(2).
25. Kocsard E. Kaposi sarcoma in a Chinese boy (aged 16 years) with localisation on the left lower extremity and on the right caruncula lacrimalis. Dermatologica. 1949;99(1):43-48.

26. Wabinga HR, Parkin DM, Wabwire-Mangen F, Nambooze S. Trends in cancer incidence in Kyadondo County, Uganda, 1960-1997. Br J Cancer. 2000;82(9):1585-1592.

27. Kaminer B, Murray JF. Sarcoma idiopathicum multiplex haemorrhagicum of Kaposi, with special reference to its incidence in the South African Negro, and two case reports. S Afr J Clin Sci. 1950;1(1):1-25.

28. Grulich AE, Beral V, Swerdlow AJ. Kaposi's sarcoma in England and Wales before the AIDS epidemic. Br J Cancer. 1992;66(6):1135-1137.

29. Geddes M, Franceschi S, Barchielli A, et al. Kaposi's sarcoma in Italy before and after the AIDS epidemic. Br J Cancer. 1994;69(2):333-336.

30. Dollard SC, Butler LM, Jones AM, et al. Substantial regional differences in human herpesvirus 8 seroprevalence in sub-Saharan Africa: insights on the origin of the "Kaposi's sarcoma belt". Int J Cancer. 2010;127(10):2395-2401

31. Cook-Mozaffari P, Newton R, Beral V, Burkitt DP. The geographical distribution of Kaposi's sarcoma and of lymphomas in Africa before the AIDS epidemic. Br J Cancer. 1998;78(11):1521-1528.

32. Rees CA, Keating EM, Lukolyo H, et al. Mapping the epidemiology of Kaposi sarcoma and non-Hodgkin lymphoma among children in subSaharan Africa: a review. Pediatr Blood Cancer. 2016;63(8):1325-1331.

33. Rohner E, Valeri F, Maskew M, et al. Incidence rate of Kaposi sarcoma in HIV-infected patients on antiretroviral therapy in Southern Africa: a prospective multicohort study. J Acquir Immune Defic Syndr. 2014;67(5):547-554.

34. Parkin DM, Sitas F, Chirenje M, Stein L, Abratt R, Wabinga H. Part I: Cancer in indigenous Africans - burden, distribution, and trends. Lancet Oncol. 2008;9(7):683-692.

35. Butler LM, Were WA, Balinandi S, et al. Human herpesvirus 8 infection in children and adults in a population-based study in rural Uganda. J Infect Dis. 2011;203(5):625-634.

36. Pfeiffer RM, Wheeler WA, Mbisa G, et al. Geographic heterogeneity of prevalence of the human herpesvirus 8 in sub-Saharan Africa: clues about etiology. Ann Epidemiol. 2010;20(12):958-963.

37. Dedicoat M, Newton R. Review of the distribution of Kaposi's sarcomaassociated herpesvirus (KSHV) in Africa in relation to the incidence of Kaposi's sarcoma. Br J Cancer. 2003;88(1):1-3.

38. Chang Y, Cesarman E, Pessin MS, et al. Identification of herpesviruslike DNA sequences in AIDS-associated Kaposi's sarcoma. Science. 1994;266(5192):1865-1869.

39. Crabtree KL, Wojcicki JM, Minhas V, Kankasa C, Mitchell C, Wood C. Association of household food- and drink-sharing practices with human herpesvirus 8 seroconversion in a cohort of Zambian children. J Infect Dis. 2017;216(7):842-849.

40. Wakeham K, Webb EL, Sebina I, et al. Risk factors for seropositivity to Kaposi sarcoma-associated herpesvirus among children in Uganda. J Acquir Immune Defic Syndr. 2013;63(2):228-233.

41. Anderson LA, Li Y, Graubard BI, et al. Human herpesvirus 8 seroprevalence among children and adolescents in the United States. Pediatr Infect Dis J. 2008;27(7):661-664.

42. Butler LM, Dorsey G, Hladik W, et al. Kaposi sarcoma-associated herpesvirus (KSHV) seroprevalence in population-based samples of African children: evidence for at least 2 patterns of KSHV transmission. J Infect Dis. 2009;200(3):430-438.

43. El-Mallawany NK, Wasswa P, Mtete I, et al. Identifying opportunities to bridge disparity gaps in curing childhood cancer in Malawi: malignancies with excellent curative potential account for the majority of diagnoses. Pediatr Hematol Oncol. 2017;34(5):261-274.

44. Mittermayer-Vassallo K, Banda K, Molyneux EM. Kaposi sarcoma in HIV-seronegative children presenting to the paediatric oncology ward in The Queen Elizabeth Central Hospital, Blantyre, Malawi during 2002-2014. Trop Doct. 2016;46(3):138-142.

45. Jackson CC, Dickson MA, Sadjadi M, et al. Kaposi sarcoma of childhood: inborn or acquired immunodeficiency to oncogenic HHV-8. Pediatr Blood Cancer. 2016;63(3):392-397. 
46. Mbulaiteye SM, Katabira ET, Wabinga H, et al. Spectrum of cancers among HIV-infected persons in Africa: the Uganda AIDS-Cancer Registry Match Study. Int J Cancer. 2006;118(4):985-990.

47. Chintu C, Athale UH, Patil PS. Childhood cancers in Zambia before and after the HIV epidemic. Arch Dis Child. 1995;73(2):100-104; discussion 104-105.

48. Athale UH, Patil PS, Chintu C, Elem B. Influence of HIV epidemic on the incidence of Kaposi's sarcoma in Zambian children. J Acquir Immune Defic Syndr Hum Retrovirol. 1995;8(1):96-100.

49. Dryden-Peterson S, Medhin H, Kebabonye-Pusoentsi M, et al. Cancer incidence following expansion of HIV treatment in Botswana. PloS One. 2015;10(8):e0135602.

50. Parkin DM, Nambooze S, Wabwire-Mangen F, Wabinga HR. Changing cancer incidence in Kampala, Uganda, 1991-2006. Int J Cancer 2010;126(5):1187-1195.

51. El-Mallawany NK, Villiera J, Kamiyango W, et al. Increasing numbers of new Kaposi sarcoma diagnoses in HIV-infected children and adolescents despite the wide availability of antiretroviral therapy in Malawi. Clin Infect Dis. 2017;64(6):818-819.

52. Siegel DA, Henley SJ, Li J, Pollack LA, Van Dyne EA, White A. Rates and trends of pediatric acute lymphoblastic leukemia - United States, 2001-2014. MMWR Morb Mortal Wkly Rep. 2017;66(36):950-954.

53. Antman K, Chang Y. Kaposi's sarcoma. N Engl J Med. 2000;342(14): 1027-1038.

54. Hengge UR, Ruzicka T, Tyring SK, et al. Update on Kaposi's sarcoma and other HHV8 associated diseases. Part 1: epidemiology, environmental predispositions, clinical manifestations, and therapy. Lancet Infect Dis. 2002;2(5):281-292.

55. El-Mallawany NK, Kamiyango W, Villiera J, et al. Proposal of a riskstratification platform to address distinct clinical features of pediatric Kaposi sarcoma in Lilongwe, Malawi. J Glob Oncol. 2017:JGO1700054.

56. Herce ME, Kalanga N, Wroe EB, et al. Excellent clinical outcomes and retention in care for adults with HIV-associated Kaposi sarcoma treated with systemic chemotherapy and integrated antiretroviral therapy in rural Malawi. J Int AIDS Soc. 2015;18:19929.

57. Venkataraman G, Uldrick TS, Aleman K, et al. Bone marrow findings in HIV-positive patients with Kaposi sarcoma herpesvirus-associated multicentric Castleman disease. Am J Clin Pathol. 2013;139(5):651-661.

58. Uldrick TS, Wang V, O'Mahony D, et al. An interleukin-6-related systemic inflammatory syndrome in patients co-infected with Kaposi sarcoma-associated herpesvirus and HIV but without Multicentric Castleman disease. Clin Infect Dis. 2010;51(3):350-358.

59. Polizzotto MN, Uldrick TS, Hu D, Yarchoan R. Clinical manifestations of Kaposi sarcoma herpesvirus lytic activation: multicentric Castleman disease (KSHV-MCD) and the KSHV inflammatory cytokine syndrome. Front Microbiol. 2012;3:73.

60. Bhutani M, Polizzotto MN, Uldrick TS, Yarchoan R. Kaposi sarcomaassociated herpesvirus-associated malignancies: epidemiology, pathogenesis, and advances in treatment. Semin Oncol. 2015;42(2):223-246.

61. Anglemyer A, Agrawal AK, Rutherford GW. Treatment of Kaposi sarcoma in children with HIV-1 infection. Cochrane Database Syst Rev. 2014;(1):CD009826.

62. Bower M, Weir J, Francis N, et al. The effect of HAART in 254 consecutive patients with AIDS-related Kaposi's sarcoma. AIDS. 2009;23(13):1701-1706.

63. Bower M, Dalla Pria A, Coyle C, et al. Prospective stage-stratified approach to AIDS-related Kaposi's sarcoma. J Clin Oncol. 2014;32(5): 409-414.

64. Krown SE. Treatment strategies for Kaposi sarcoma in sub-Saharan Africa: challenges and opportunities. Curr Opin Oncol. 2011;23(5):463-468.
65. Krown SE, Borok MZ, Campbell TB, et al. Stage-stratified approach to AIDS-related Kaposi's sarcoma: implications for resource-limited environments. J Clin Oncol. 2014;32(23):2512-2513.

66. Campbell L, Bacha J, Slone JS, El-Mallawany NK, Mwita L, Mehta PS. Characteristics and outcomes of pediatric Kaposi sarcoma patients in the southern highlands zone of Tanzania. Paper presented at: 10th International Conference on Cancer in Africa (AORTIC); November 20, 2015; Marrakech, Morocco.

67. Letang E, Lewis JJ, Bower M, et al. Immune reconstitution inflammatory syndrome associated with Kaposi sarcoma: higher incidence and mortality in Africa than in the UK. AIDS. 2013;27(10):1603-1613.

68. Mwafongo AA, Rosenberg NE, Ng'ambi W, et al. Treatment outcomes of AIDS-associated Kaposi's sarcoma under a routine antiretroviral therapy program in Lilongwe, Malawi: bleomycin/vincristine compared to vincristine monotherapy. PloS One. 2014;9(3):e91020.

69. Goncalves PH, Ziegelbauer J, Uldrick TS, Yarchoan R. Kaposi sarcoma herpesvirus-associated cancers and related diseases. Curr Opin HIV AIDS. 2017;12(1):47-56.

70. Dittmer DP, Damania B. Kaposi sarcoma-associated herpesvirus: immunobiology, oncogenesis, and therapy. J Clin Invest. 2016;126(9):3165-3175.

71. Chadburn A, Said J, Gratzinger D, et al. HHV8/KSHV-positive lymphoproliferative disorders and the spectrum of plasmablastic and plasma cell neoplasms: $2015 \mathrm{SH}$ /EAHP workshop report-part 3. Am J Clin Pathol. 2017;147(2):171-187.

72. Mylona EE, Baraboutis IG, Lekakis LJ, Georgiou O, Papastamopoulos V, Skoutelis A. Multicentric Castleman's disease in HIV infection: a systematic review of the literature. AIDS Rev. 2008;10(1):25-35.

73. Polizzotto MN, Uldrick TS, Wyvill KM, et al. Clinical features and outcomes of patients with symptomatic Kaposi sarcoma herpesvirus (KSHV)-associated inflammation: prospective characterization of KSHV inflammatory cytokine syndrome (KICS). Clin Infect Dis. 2016;62(6):730-738.

74. Goncalves PH, Uldrick TS, Yarchoan R. HIV-associated Kaposi sarcoma and related diseases. AIDS. 2017;31(14):1903-1916.

75. Kaplan LD. Human herpesvirus-8: Kaposi sarcoma, multicentric Castleman disease, and primary effusion lymphoma. Hematology Am Soc Hematol Educ Program. 2013;2013:103-108.

76. Sakakibara S, Tosato G. Viral interleukin-6: role in Kaposi's sarcomaassociated herpesvirus: associated malignancies. J Interferon Cytokine Res. 2011;31(11):791-801.

77. Moore PS, Boshoff C, Weiss RA, Chang Y. Molecular mimicry of human cytokine and cytokine response pathway genes by KSHV. Science. 1996;274(5293):1739-1744.

78. Polizzotto MN, Uldrick TS, Wang V, et al. Human and viral interleukin-6 and other cytokines in Kaposi sarcoma herpesvirus-associated multicentric Castleman disease. Blood. 2013;122(26):4189-4198.

79. Gopal S, Fedoriw Y, Montgomery ND, et al. Multicentric Castleman's disease in Malawi. Lancet. 2014;384(9948):1158.

80. Hosseinipour MC, Sweet KM, Xiong J, et al. Viral profiling identifies multiple subtypes of Kaposi's sarcoma. MBio. 2014;5(5): e01633-01714.

81. Miller H, Slone JS, Raabe E, El-Mallawany NK, Mehta P, Phelps BR. Lessons from pediatric HIV: a case for curative intent in pediatric cancer in LMICs. Pediatrics. 2017;140(4).

82. de Amicis T. Studio clinico ed anatomo-patologico su dudici nuove osservazioni di dermo-polimelano-sarcoma idiopatico. Tipografia A. Trani. [Clinical and anatomy-pathological study on new dermatological observations of idiopathic dermo-polimelano-sarcoma. A. Trani typography]. Naples, Italy: 1882. Italian. 


\section{Publish your work in this journal}

Pediatric Health, Medicine and Therapeutics is an international, peerreviewed, open access journal publishing original research, reports, editorials, reviews and commentaries. All aspects of health maintenance, preventative measures and disease treatment interventions are addressed within the journal. Practitioners from all disciplines are invited to submit their work as well as healthcare researchers and patient support groups. The manuscript management system is completely online and includes a very quick and fair peer-review system. Visit http://www.dovepress.com/ testimonials.php to read real quotes from published authors.

Submit your manuscript here: http://www.dovepress.com/pediatric-health-medicine-and-therapeutics-journal 